\title{
Heating of accretion-disk coronae and jets by general relativistic magnetohydrodynamic turbulence
}

\author{
Benjamin D. G. Chandran ${ }^{1, \dagger}$, Francois Foucart ${ }^{1,2}$ and \\ Alexander Tchekhovskoy ${ }^{2,3,4}$ \\ ${ }^{1}$ Department of Physics, University of New Hampshire, Durham, NH 03824, USA \\ ${ }^{2}$ Lawrence Berkeley National Laboratory, 1 Cyclotron Rd, Berkeley, CA 94720, USA \\ ${ }^{3}$ Departments of Astronomy and Physics, Theoretical Astrophysics Center, University of California \\ Berkeley, Berkeley, CA 94720-3411, USA \\ ${ }^{4}$ Center for Interdisciplinary Exploration \& Research in Astrophysics (CIERA), Physics \& Astronomy, \\ Northwestern University, Evanston, IL 60202, USA
}

(Received 16 October 2017; revised 14 April 2018; accepted 16 April 2018)

Turbulence in an accretion disk launches Alfvén waves (AWs) that propagate away from the disk along magnetic-field lines. Because the Alfvén speed varies with distance from the disk, the AWs undergo partial non-WKB reflection, and counter-propagating AWs subsequently interact, causing AW energy to cascade to small scales and dissipate. To investigate this process, we introduce an Elsasser-like formulation of general relativistic magnetohydrodynamics (GRMHD) and develop the theory of general relativistic reduced MHD in an inhomogeneous medium. We then derive a set of equations for the mean-square AW amplitude $M_{+}$and turbulent heating rate $Q$ under the assumption that, in the plasma rest frame, AWs propagating away from the disk are much more energetic than AWs propagating toward the disk. For the case in which the background flow is axisymmetric and time independent, we solve these equations analytically to determine $M_{+}$and $Q$ as functions of position. We find that, for an idealized thin disk threaded by a large-scale poloidal magnetic field, the AW energy flux is $\sim\left(\rho_{\mathrm{b}} / \rho_{\mathrm{d}}\right)^{1 / 2} \beta_{\text {net,d }}^{-1 / 2}$ times the disk's radiative flux, where $\rho_{\mathrm{b}}$ and $\rho_{\mathrm{d}}$ are the mass densities at the coronal base and disk midplane, respectively, and $\beta_{\text {net,d }}$ is the ratio (evaluated at the disk midplane) of plasma-plus-radiation pressure to the pressure of the average vertical magnetic field. This energy flux could have a significant impact on disk coronae and outflows. To lay the groundwork for future global simulations of turbulent disk coronae and jets, we derive a set of averaged GRMHD equations that account for reflection-driven AW turbulence using a sub-grid model.

Key words: astrophysical plasmas, plasma nonlinear phenomena, space plasma physics 


\section{Introduction}

Several types of evidence, including the observed velocities of stars (Ghez et al. 2005) and gas (Miyoshi et al. 1995) near galactic centres and gravitational-wave signals indicative of black-hole (BH) mergers (Abbott et al. 2016), reveal BHs scattered throughout the visible universe with masses ranging from several to billions of solar masses. As a BH pulls in plasma from its surroundings, the plasma's angular momentum causes the inflowing plasma to form a disk. If this accretion disk is geometrically thin but optically thick ('a thin disk'), then turbulent viscosity converts a significant fraction of the plasma's gravitational potential energy into thermal energy that is radiated away before the material reaches the central $\mathrm{BH}$. In part because of this, thin disks are promising candidates for explaining much of the continuum emission from high-luminosity active galactic nuclei (AGN) and stellar-mass BHs in binary systems in their brighter states (Novikov \& Thorne 1973; Shakura \& Sunyaev 1973). Disks that are geometrically thick but optically thin ('thick disks') are generally much less luminous than thin disks, because plasma in the disk can fall into the $\mathrm{BH}$ before it radiates much of its thermal energy (Narayan \& Yi 1994), become marginally unstable to convection, which suppresses mass inflow to the central BH (Quataert \& Gruzinov 2000), or become gravitationally unbound and flow outward (Blandford \& Begelman 1999). Thick disks are thought to be present around low-luminosity BHs, such as Sagittarius $A^{*}$ at the centre of our galaxy (Narayan \& Yi 1994; Quataert \& Gruzinov 2000).

BH/accretion-disk systems launch two types of outflows: non-collimated winds, which can be mildly relativistic or non-relativistic, and collimated, relativistic jets. The jets emanating from BH systems at the centres of galaxies are particularly striking, because they can span hundreds of kiloparsecs (Fanaroff \& Riley 1974). Theoretical studies (e.g. Blandford \& Znajek 1977) and numerical simulations (De Villiers, Hawley \& Krolik 2003a; McKinney \& Gammie 2004; Tchekhovskoy, Narayan \& McKinney 2011) have identified a promising mechanism for producing jets via a large-scale, ordered magnetic field that threads an accretion disk or the event horizon of a rotating $\mathrm{BH}$. The rotation of the disk or $\mathrm{BH}$ coils up the magnetic-field lines, which then act as a spring, pushing material away from the disk along the spin axis.

Although this mechanism offers an explanation for jet formation and acceleration, it is not yet clear how the mass outflow rates and mechanical luminosities of jets and winds are determined. Nor is it clear what accelerates the particles that cause a jet, or the plasma at a jet's base, to radiate. For example, it is unclear how to account for X-ray timing observations that indicate that many luminous AGN contain compact coronae - i.e. high-temperature, optically thin plasma - within a few gravitational radii of the central BH (Reis \& Miller 2013).

Clues to these puzzles may be offered by a system much closer to home. In one explanation for the heating and acceleration of the solar wind, convection-driven photospheric motions shake the footpoints of 'open' magnetic-field lines (i.e. field lines that connect directly to the interplanetary medium). This shaking launches Alfvén waves (AWs) that propagate along the magnetic-field lines, through coronal holes (open-field regions of the corona), and into the solar wind (Cranmer \& van Ballegooijen 2005). Because the Alfvén speed varies with distance from the Sun, these outward-propagating AWs undergo partial non-WKB (Wentzel-Kramers-Brillouin) reflection (Heinemann \& Olbert 1980; Velli 1993). Counter-propagating AWs subsequently interact, which causes the AWs to become turbulent, which in turn causes AW energy to cascade from large wavelengths to small wavelengths and dissipate, heating the ambient plasma. This heating increases the plasma pressure, 
which, along with the wave pressure, accelerates the solar wind to supersonic speeds. This explanation for the solar wind's origin is supported by numerous observational, theoretical and numerical studies (e.g. Cranmer \& van Ballegooijen 2005; Cranmer, van Ballegooijen \& Edgar 2007; De Pontieu et al. 2007; Verdini \& Velli 2007; Hollweg, Cranmer \& Chandran 2010; Verdini et al. 2010; Chandran et al. 2011; Perez \& Chandran 2013; van der Holst et al. 2014; Usmanov, Goldstein \& Matthaeus 2014; van Ballegooijen \& Asgari-Targhi 2016, 2017). Turbulence plays a key role in this model, because the large-wavelength AWs launched by the Sun are damped so weakly that, without turbulence, they would reach the distant interplanetary medium without appreciably damping or heating the plasma (Barnes 1966). Wave reflection is a critical component of the model because the Sun launches only outward-propagating waves, and AWs interact to produce turbulence only when there is a mix of counter-propagating AWs in the plasma rest frame (Iroshnikov 1963; Kraichnan 1965).

In this paper, we explore the possibility that similar physical processes contribute to the generation of accretion-disk coronae and jets. In particular, we consider the fate of AWs that are launched by a turbulent accretion disk into the disk's corona and an overlying outflow. To allow for space-time curvature, relativistic fluid velocities, relativistic Alfvén speeds and relativistic thermal velocities, we work within the framework of general relativistic magnetohydrodynamics (GRMHD). Previous studies have investigated the heating of accretion-disk coronae by the reconnection of magnetic loop structures (e.g. Galeev, Rosner \& Vaiana 1979; Uzdensky \& Goodman 2008). Our work focuses on AW turbulence rather than magnetic reconnection, and open-field regions rather than closed magnetic loops.

The remainder of this paper is organized as follows. In $\S 2$ we derive a set of equations that describes AW propagation, reflection and nonlinear interactions in an inhomogeneous background flow. In $\S 3$ we specialize to the case of a time-independent and axisymmetric background and solve analytically for the mean-square AW amplitude and turbulent heating rate as functions of position. In $\S 4$ we apply our results to the corona and outflow overlying a thin accretion disk in the $\alpha$-disk model (Novikov \& Thorne 1973; Shakura \& Sunyaev 1973). In $\S 5$ we derive a set of averaged GRMHD equations in which AW turbulence is treated using a sub-grid model. These equations complement the results of $\S 2$ by describing how AW turbulence influences the background flow via turbulent heating and momentum deposition.

\section{Reflection-driven Alfvén-wave turbulence in general relativity}

GRMHD describes a highly conducting magnetized fluid under the assumption that the Lorentz force vanishes for a charged particle at rest in the local plasma frame. This assumption simplifies the source-free subset of Maxwell's equations and the electromagnetic contribution to the stress-energy tensor (see, e.g. Anile 1989; Gammie, McKinney \& Tóth 2003). A GRMHD fluid is described by the equation of mass conservation,

$$
\left(\rho u^{\nu}\right)_{; \nu}=0,
$$

the stress-energy equation,

$$
T_{; \nu}^{\mu \nu}=0,
$$

and the relativistic induction equation,

$$
\left(b^{\mu} u^{\nu}-b^{v} u^{\mu}\right)_{; \nu}=0,
$$


where $\rho$ is the mass density, $u^{\mu}$ is the 4-velocity,

$$
T^{\mu \nu}=\mathcal{E} u^{\mu} u^{\nu}+\left(p+\frac{b^{2}}{2}\right) g^{\mu \nu}-b^{\mu} b^{\nu}
$$

is the GRMHD stress-energy tensor,

$$
b^{\mu}=\frac{1}{2} \epsilon^{\mu \nu \kappa \lambda} u_{\nu} F_{\lambda \kappa}
$$

is the magnetic-field 4-vector, $b^{2}=b^{\mu} b_{\mu}, F_{\lambda \kappa}$ is the Faraday tensor divided by $\sqrt{4 \pi}$, $\epsilon^{\mu \nu \kappa \lambda}$ is the Levi-Civita tensor,

$$
\mathcal{E}=\rho+u+p+b^{2}
$$

$u$ (without indices) is the internal energy, $p$ is the pressure, $g_{\mu \nu}$ is the metric tensor and the units have been chosen so that the speed of light is 1 (Komissarov 1999; Gammie et al. 2003). The semicolon subscripts indicate covariant differentiation, repeated indices are summed and Greek indices range from 0 to 3. The 4-velocity satisfies

$$
u^{\mu} u_{\mu}=-1
$$

and it follows from (2.5) that

$$
u_{\mu} b^{\mu}=0
$$

The magnetic-field 3-vector is given by

$$
B^{i}=b^{i} u^{t}-b^{t} u^{i}
$$

where Latin indices range from 1 to 3 , and $t$ indices indicate the time component. Equation (2.9) can be inverted using (2.7) and (2.8) to give (Gammie et al. 2003)

$$
b^{t}=B^{i} u^{\mu} g_{i \mu} \quad b^{i}=\frac{B^{i}+b^{t} u^{i}}{u^{t}} .
$$

Equation (2.3) can then be rewritten as the two equations

$$
\frac{1}{\sqrt{-g}} \partial_{i}\left(\sqrt{-g} B^{i}\right)=0
$$

and

$$
\partial_{t}\left(\sqrt{-g} B^{i}\right)=\partial_{j}\left[\sqrt{-g}\left(B^{j} v^{i}-B^{i} v^{j}\right)\right],
$$

where $\partial_{\mu}$ indicates differentiation with respect to coordinate $\mu, g$ is the determinant of the metric tensor and $v^{i}=u^{i} / u^{t}$ is the fluid 3-velocity (Gammie et al. 2003). As described in $\S 5, b^{\mu}$ is the magnetic field in the fluid frame (in the sense that is explained prior to (5.4)), while $B^{i}$ is the 'laboratory-frame' magnetic field when $g^{t t}=$ -1 . 


\subsection{An Elsasser-like formulation of GRMHD}

Elsasser (1950) reformulated non-relativistic MHD, obtaining a set of equations that is useful for studying AWs and AW turbulence. We obtain an Elasser-like formulation of GRMHD by multiplying (2.3) by $\pm \mathcal{E}^{1 / 2}$, adding the resulting expression to (2.2) and then dividing by $\mathcal{E}$. This yields

$$
\left(z_{ \pm}^{\mu} z_{\mp}^{\nu}+\Pi g^{\mu \nu}\right)_{; \nu}+\left(\frac{3}{4} z_{ \pm}^{\mu} z_{\mp}^{\nu}+\frac{1}{4} z_{\mp}^{\mu} z_{ \pm}^{\nu}+\Pi g^{\mu \nu}\right) \frac{\partial_{\nu} \mathcal{E}}{\mathcal{E}}=0
$$

where

$$
z_{ \pm}^{\mu}=u^{\mu} \mp \frac{b^{\mu}}{\mathcal{E}^{1 / 2}} \quad \Pi=\frac{2 p+b^{2}}{2 \mathcal{E}}
$$

\subsection{Background quantities, fluctuations and the average fluid rest frame}

We assume that each property of the fluid is the sum of a smoothly varying background value plus a fluctuation,

$$
u^{\mu}=\overline{u^{\mu}}+\delta u^{\mu} \quad b^{\mu}=\overline{b^{\mu}}+\delta b^{\mu} \quad \text { etc. }
$$

We construct an 'average fluid rest frame' (AFRF) at each point by first transforming to locally Galilean coordinates and then carrying out a Lorentz transformation that causes $\overrightarrow{u^{i}}$ to vanish while leaving the metric in Minkowski form at that point. We define $\lambda$ to be the perpendicular correlation length (i.e. the correlation length measured perpendicular to $\overline{B^{i}}$ ) of the velocity and magnetic-field fluctuations in the AFRF, and $L$ to be the characteristic length scale of the background quantities and $g_{\mu \nu}$ in the AFRF. We assume that

$$
\lambda / L \sim O(\epsilon)
$$

where $\epsilon$ (without indices) is a small parameter. We use the notation $\langle\ldots\rangle$ to denote a volume average within a sphere of radius $d$ in the AFRF, with $\lambda \ll d \ll L$. For any vector $f^{\mu}$, we assume that the following assertions lead to negligible error: $\left\langle f^{\mu}\right\rangle$ is a vector, $\left\langle\left\langle f^{\mu}\right\rangle\right\rangle=\left\langle f^{\mu}\right\rangle=\overline{f^{\mu}}$ and $\left\langle f_{; \nu}^{\mu}\right\rangle=\left\langle f^{\mu}\right\rangle_{; \nu}$, with analogous statements for scalars and tensors. We note that $\overline{u^{\mu}}$ is not a unit vector in the sense of (2.7) and is therefore not a 4-velocity. It is merely the local spatial average, in the AFRF, of $u^{\mu}$. The 4-velocity of the AFRF is given in (5.1) below.

\subsection{General relativistic reduced MHD in an inhomogeneous background}

To motivate the next step in our analysis, we return for a moment to the solar analogy. Spacecraft measurements indicate that $B^{i}$ and $v^{i}$ fluctuations in the solar wind are mostly transverse (orthogonal to $\overline{B^{i}}$ ) and non-compressive (Klein, Roberts \& Goldstein 1991; Horbury et al. 1995; Tu \& Marsch 1995). One reason for this is that the dominant dissipation mechanism for slow magnetosonic modes and entropy modes, turbulent mixing, causes the energy of these compressive modes to decay on the time scale $\lambda / \delta u_{\mathrm{rms}}$, where $\delta u_{\mathrm{rms}}$ is the root-mean-square (r.m.s.) amplitude of the velocity fluctuations (Schekochihin et al. 2016). This time scale is shorter than the energy-decay time scale for outward-propagating, 
non-compressive, AW fluctuations, which is $\sim \lambda / \delta u_{\text {inward }}$ (Iroshnikov 1963; Kraichnan 1965), where $\delta u_{\text {inward }}$ is the r.m.s. velocity fluctuation of the inward-propagating AWs. The inequality $\lambda / \delta u_{\mathrm{rms}} \ll \lambda / \delta u_{\text {inward }}$ follows from in situ measurements (Bavassano, Pietropaolo \& Bruno 2000) and numerical models (Cranmer \& van Ballegooijen 2005; Verdini \& Velli 2007; Perez \& Chandran 2013; van Ballegooijen \& Asgari-Targhi 2016) that show that outward-propagating AWs have much larger amplitudes than inward-propagating AWs in the near-Sun solar wind. The other compressive MHD mode, the fast magnetosonic mode, has an even smaller amplitude in the solar wind than the slow magnetosonic mode (Yao et al. 2011; Howes et al. 2012; Klein et al. 2012), in part because fast magnetosonic waves launched by the Sun are reflected back towards the Sun by the rapid increase in $v_{\mathrm{A}}$ between the chromosphere and corona (Hollweg 1978).

We conjecture that turbulence in jets and disk coronae is mostly transverse and non-compressive (AW-like) for similar reasons. We thus consider just the AW-like component of the turbulence by adopting the orderings of reduced MHD (RMHD),

$$
\left(\delta u^{2}\right)^{1 / 2} \equiv\left(\delta u^{\mu} \delta u_{\mu}\right)^{1 / 2} \sim\left(\frac{\delta b^{\mu} \delta b_{\mu}}{\mathcal{E}}\right)^{1 / 2} \sim O\left(\epsilon v_{\mathrm{A}}\right)
$$

and

$$
\delta \rho / \bar{\rho} \sim \delta u / \bar{u} \sim \delta p / \bar{p} \sim O\left(\epsilon^{2}\right),
$$

where

$$
v_{\mathrm{A}}^{\mu} \equiv \overline{b^{\mu}} / \overline{\mathcal{E}}^{1 / 2} \quad v_{\mathrm{A}}=\left(v_{\mathrm{A}}^{\mu} v_{\mathrm{A} \mu}\right)^{1 / 2},
$$

and by adopting the RMHD assumption that in the AFRF $\delta z_{ \pm i} \overline{B^{i}}=0$ and $\partial_{i} \delta u^{i}=$ 0 . Equations (2.7) and (2.8) and their averages imply that, in the AFRF, $\mathcal{E}^{-1 / 2} \overline{b_{t}} \sim$ $\mathcal{E}^{-1 / 2} \delta b_{t} \sim \delta u_{t} \sim O\left(\epsilon^{2} v_{\AA}^{2}\right)$, from which it follows that

$$
\overline{z_{ \pm}^{\mu}} \delta z_{ \pm \mu} \sim \overline{z_{\mp}^{\mu}} \delta z_{ \pm \mu} \sim O\left(\epsilon^{2} v_{\mathrm{A}}^{2}\right) \quad \delta z_{ \pm ; \nu}^{\nu} \sim O\left(\epsilon v_{\mathrm{A}} / L\right) .
$$

As in non-relativistic RMHD (see, e.g. Schekochihin et al. 2009), we take the parallel correlation length (i.e. the correlation length measured parallel to $\overline{B^{i}}$ ) of $\delta z_{ \pm}^{\mu}$ in the AFRF to be $\sim O(\lambda / \epsilon) \sim O(L)$, and thus

$$
\overline{z_{\mp}^{v}} \partial_{\nu} \delta z_{ \pm}^{\mu} \sim \delta z_{\mp}^{v} \partial_{\nu} \delta z_{ \pm}^{\mu} \sim O\left(v_{\mathrm{A}} \delta z_{ \pm}^{\mu} / L\right)
$$

Subtracting the average of (2.13) from (2.13) and dropping terms $\ll \delta z_{ \pm}^{\mu} v_{\mathrm{A}} / L$, we obtain

$$
\left(\delta z_{ \pm}^{\mu} \overline{z_{\mp}^{v}}+\overline{z_{ \pm}^{\mu}} \delta z_{\mp}^{v}\right)_{; \nu}+\left(\frac{3}{4} \delta z_{ \pm}^{\mu} \overline{z_{\mp}^{v}}+\frac{3}{4} \overline{z_{ \pm}^{\mu}} \delta z_{\mp}^{\nu}+\frac{1}{4} \delta z_{\mp}^{\mu} \overline{z_{ \pm}^{v}}+\frac{1}{4} \overline{z_{\mp}^{\mu}} \delta z_{ \pm}^{v}\right) \frac{\partial_{\nu} \overline{\mathcal{E}}}{\overline{\mathcal{E}}}=-N_{ \pm}^{\mu},
$$

where

$$
N_{ \pm}^{\mu}=\left(\delta z_{ \pm}^{\mu} \delta z_{\mp}^{\nu}+\delta \Pi g^{\mu \nu}\right)_{; \nu} .
$$

The nonlinear $\left(\delta z_{ \pm}^{\mu} \delta z_{\mp}^{v}\right)_{; \nu}$ term in $N_{ \pm}^{\mu}$ is non-zero only in the presence of both $\delta z_{+}^{\mu}$ and $\delta z_{-}^{v}$ fluctuations, implying that nonlinear interactions arise only between counterpropagating AW packets, as in the non-relativistic limit (Iroshnikov 1963; Kraichnan 1965). We assume that, as in non-relativistic RMHD, the role of the $\delta \Pi$ term in $N_{ \pm}^{\mu}$ is merely to cancel out the compressive component of the nonlinear term in the AFRF (Maron \& Goldreich 2001). 


\subsection{Reflection-driven GRMHD turbulence}

We take the fluctuations to be statistically gyrotropic in the AFRF, which, given (2.20), implies that

$$
\left\langle\delta z_{ \pm}^{\mu} \delta z_{ \pm}^{\nu}\right\rangle=\frac{1}{2} M_{ \pm}\left(g^{\mu \nu}+\overline{u^{\mu}} \overline{u^{\nu}}-\frac{\overline{b^{\mu}} \overline{b^{v}}}{b^{2}}\right)
$$

and

$$
\left\langle\delta z_{+}^{\mu} \delta z_{-}^{\nu}\right\rangle=\frac{1}{2} R\left(g^{\mu \nu}+\overline{u^{\mu}} \overline{u^{\nu}}-\frac{\overline{b^{\mu}} \overline{b^{\nu}}}{b^{2}}\right),
$$

where $M_{ \pm}$and $R$ are scalars. The quantity $\delta z_{ \pm}^{\mu}$ corresponds to AWs that propagate in the AFRF either parallel or anti-parallel to the background magnetic field. Because an accretion disk launches only outward-propagating fluctuations, we assume in what follows that outward-propagating $\mathrm{AWs}\left(\delta z_{+}^{\mu}\right.$, for concreteness) have much larger amplitudes than inward-propagating $\operatorname{AWs}\left(\delta z_{-}^{\mu}\right)$,

$$
M_{+} \gg M_{-} \quad M_{+} \gg R,
$$

as in the near-Sun solar wind and coronal holes (Bavassano et al. 2000; Cranmer \& van Ballegooijen 2005). We then drop terms that are $\ll \delta z_{+}^{\mu} v_{\mathrm{A}} / L$ in (2.22) to obtain

$$
\left(\delta z_{+}^{\mu} \overline{z_{-}^{v}}\right)_{; \nu}+\left(\frac{3}{4} \delta z_{+}^{\mu} \overline{z_{-}^{v}}+\frac{1}{4} \overline{z_{-}^{\mu}} \delta z_{+}^{\nu}\right) \frac{\partial_{\nu} \overline{\mathcal{E}}}{\overline{\mathcal{E}}}=-N_{+}^{\mu} .
$$

For future reference, when $M_{+} \gg M_{-}$, to a good approximation $\delta u^{\mu}=-\delta b^{\mu} / \overline{\mathcal{E}}^{1 / 2}$, $\delta z_{+}^{\mu}=2 \delta u^{\mu}$, and

$$
\left\langle\delta u^{2}\right\rangle=\frac{M_{+}}{4} .
$$

Motivated by models of solar-wind turbulence that were reasonably successful at explaining observations (Chandran \& Hollweg 2009; Chandran et al. 2011), we approximate $N_{ \pm}^{\mu}$ as a nonlinear damping term (Dmitruk et al. 2002), setting

$$
N_{ \pm}^{\mu}=\gamma_{ \pm} \delta z_{ \pm}^{\mu} \quad \gamma_{ \pm}=\frac{c_{1} \sqrt{M_{\mp}}}{\lambda}
$$

where $c_{1}$ is some constant of order unity. In taking $\gamma_{ \pm}$to be $\propto \sqrt{M_{\mp}}$, we have made use of the fact that $\delta z_{ \pm}^{\mu}$ fluctuations are sheared only by $\delta z_{\mp}^{\mu}$ fluctuations. Contracting (2.27) with $2 \delta z_{+\mu}$ and averaging, we obtain

$$
\overline{z_{-}^{v}} \partial_{\nu} M_{+}+M_{+}\left(2 \overline{z_{-; \nu}^{v}}+\frac{3 \overline{z_{-}^{v}} \partial_{\nu} \overline{\mathcal{E}}}{2 \overline{\mathcal{E}}}\right)=-2 \gamma_{+} M_{+} .
$$

Again dropping terms $\ll \delta z_{+}^{\mu} v_{\mathrm{A}} / L$ in (2.22), but this time choosing the lower sign, we obtain

$$
\left(\overline{z_{-}^{\mu}} \delta z_{+}^{\nu}\right)_{; \nu}+\left(\frac{3}{4} \overline{z_{-}^{\mu}} \delta z_{+}^{\nu}+\frac{1}{4} \delta z_{+}^{\mu} \overline{z_{-}^{\nu}}\right) \frac{\partial_{\nu} \overline{\mathcal{E}}}{\overline{\mathcal{E}}}=-\gamma_{-} \delta z_{-}^{\mu} .
$$

Equation (2.31) states that $\delta z_{-}^{\mu}$ is determined locally by balancing the rate at which $\delta z_{-}^{\mu}$ is produced by the reflection of $\delta z_{+}^{\mu}$ fluctuations against the rate at which $\delta z_{-}^{\mu}$ 
fluctuations are cascaded to small scales. Contracting (2.31) with $\delta z_{ \pm \mu}$ and averaging, we obtain

$$
\gamma_{-} R=\frac{M_{+} \overline{z_{-}^{v}} \partial_{\nu} v_{\mathrm{A}}}{2 v_{\mathrm{A}}} \quad \gamma_{-} M_{-}=\frac{R \overline{z_{-}^{v}} \partial_{\nu} v_{\mathrm{A}}}{2 v_{\mathrm{A}}} .
$$

Since $\gamma_{-}^{2} M_{-}=\gamma_{+}^{2} M_{+}$, equation (2.32) yields

$$
\gamma_{+}=\left|\frac{\overline{z_{-}^{v}} \partial_{\nu} v_{\mathrm{A}}}{2 v_{\mathrm{A}}}\right|
$$

which does not depend on the unknown constant $c_{1}$ introduced in (2.29).

As $\delta z_{+}^{\mu}$ fluctuations propagate away from the disk, the value of $v_{\mathrm{A}}$ at their instantaneous location keeps changing. Equations (2.30) and (2.33) imply that each time $v_{\mathrm{A}}$ changes by a factor of $\sim 2$, a modest fraction of the fluctuation energy cascades and dissipates. A substantial fraction of the AW energy launched by a disk thus dissipates within several $v_{\mathrm{A}}$ scale heights of the disk, offering a natural explanation for the compact coronae detected in high-luminosity AGN (Reis \& Miller 2013). We show in $\S 5$ that the turbulent heating rate is

$$
Q=\frac{1}{2} \gamma_{+} \overline{\mathcal{E}} M_{+}
$$

If $\overline{u^{\mu}}, \overline{v_{\mathrm{A}}^{\mu}}$, and $\overline{\mathcal{E}}$ are known, equations (2.30), (2.33) and (2.34) can be solved to determine $M_{+}$and $Q$.

\section{Reflection-driven Alfvén-wave turbulence in a stationary, axisymmetric background}

We now work in the frame of the central compact object (e.g. Boyer-Lindquist (1967) coordinates) and take averaged quantities in this frame to be independent of time and cylindrical angle $\phi$. We decompose the spatial components of $\overline{u^{\mu}}$ into poloidal and toroidal 3-vectors,

$$
\overline{u^{i}}=u_{\mathrm{p}}^{i}+u_{\mathrm{T}}^{i}
$$

and likewise for $\overline{b^{i}}, \overline{v_{\mathrm{A}}^{i}}$ and $\overline{B^{i}}$, where the poloidal vectors have vanishing $\boldsymbol{\phi}$ components and the toroidal vectors are proportional to the $\phi$ basis vector. It then follows from (2.12) that (Mestel 1961)

$$
u_{\mathrm{p}}^{i}=\kappa B_{\mathrm{p}}^{i}
$$

where $\kappa$ depends upon position. Equations (2.1), (2.11), and (3.2) imply that

$$
B_{\mathrm{p}}^{i} \partial_{i}(\bar{\rho} \kappa)=0 .
$$

With the use of (2.10), (2.19) and (3.2), we obtain

$$
b_{\mathrm{p}}^{i}=\eta B_{\mathrm{p}}^{i} \quad v_{\mathrm{Ap}}^{i}=y u_{\mathrm{p}}^{i},
$$

where

$$
\eta=\frac{1+\kappa \overline{b^{t}}}{\overline{u^{t}}} \quad y=\frac{\eta}{\kappa \overline{\mathcal{E}}^{1 / 2}}
$$


In the Appendix, we show, given (3.1) through (3.5), that (2.30) can be rewritten as

$$
\left(u_{\mathrm{p}}^{i}+v_{\mathrm{Ap}}^{i}\right) \partial_{i}\left(\chi M_{+}\right)=-2 \gamma_{+} \chi M_{+},
$$

where

$$
\chi=\frac{\overline{\mathcal{E}}^{3 / 2}\left(u_{\mathrm{p}}+v_{\mathrm{Ap}}\right)^{2}}{\bar{\rho}^{2} u_{\mathrm{p}}^{2}} .
$$

Close to the disk, $v_{\mathrm{A}}$ increases with distance from the disk, $\overline{z_{-}^{v}} \partial_{v} \ln v_{\mathrm{A}}>0$, and (2.33) and (3.6) imply that $\chi M_{+} v_{\mathrm{A}}$ is constant along a field line. Equivalently,

$$
M_{+}=M_{+\mathrm{b}}\left(\frac{\chi_{\mathrm{b}}}{\chi}\right)\left(\frac{v_{\mathrm{Ab}}}{v_{\mathrm{A}}}\right),
$$

where the subscript $b$ indicates that the subscripted quantity is evaluated at the base of the disk's corona on the magnetic-field line that passes through the observation point, at which the unsubscripted $M_{+}, \chi$, and $v_{\mathrm{A}}$ terms in (3.8) are evaluated. If $v_{\mathrm{A}}$ increases monotonically with increasing distance from the disk, then (3.8) remains valid to all distances. On the other hand, if the value of $v_{\mathrm{A}}$ along the magnetic-field line that passes through the observation point reaches a maximum value $v_{\mathrm{Am}}$ at a distance $r=$ $r_{\mathrm{m}}$ from the central $\mathrm{BH}$, then at $r>r_{\mathrm{m}}$ (2.33) and (3.6) imply that $\chi M_{+} / v_{\mathrm{A}}$ is constant along the magnetic field. Combining this result with (3.8), we find that

$$
M_{+}=M_{+\mathrm{b}}\left(\frac{\chi_{\mathrm{b}}}{\chi}\right)\left(\frac{v_{\mathrm{Ab}} v_{\mathrm{A}}}{v_{\mathrm{Am}}^{2}}\right)
$$

at $r>r_{\mathrm{m}}$. If $v_{\mathrm{A}}$ progresses through an alternating series of maxima and minima, then $M_{+}$can be found by taking $\chi M_{+} v_{\mathrm{A}}$ to be constant along a field line between each $v_{\mathrm{A}}$ minimum and the next maximum farther out, and taking $\chi M_{+} / v_{\mathrm{A}}$ to be constant between each maximum and the next minimum. Once $M_{+}$is determined, the turbulent heating rate follows from (2.33) and (2.34). Equations (3.8) and (3.9) generalize previous results on solar-wind turbulence (Dmitruk et al. 2002; Chandran \& Hollweg 2009) by allowing for curved space-time, relativistic velocities, and non-zero toroidal components and non-radial poloidal components of $\overline{B^{i}}$ and $\overline{u^{i}}$.

\section{Application to coronae and outflows above thin accretion disks}

As an example, we now apply our results to the corona and outflow above a steadystate, thin accretion disk threaded by a large-scale poloidal magnetic field. We define the coronal base to be the surface on which $\beta_{\text {total }}=1$, where

$$
\beta_{\text {total }} \equiv \frac{2\left(p+p_{\text {rad }}\right)}{B^{2}},
$$

and $p_{\text {rad }}$ is the radiation pressure. Below the coronal base (i.e. in the disk), $\beta_{\text {total }}>1$; above the coronal base, $\beta_{\text {total }}<1$. The results of $\S \S 2.4$ and 3 are based on the assumptions that $M_{+} \gg M_{-}$and that reflection is the primary source of inward-propagating AWs $\left(\delta z_{-}^{\mu}\right)$. These assumptions are reasonable above the $\beta_{\text {total }}=1$ surface, because when $\beta_{\text {total }}<1$ the magnetorotational instability (MRI) is stable at wavelengths comparable to or smaller than the disk thickness, and because the disk launches only outward-propagating waves. These assumptions, however, are not satisfied below the $\beta_{\text {total }}=1$ surface, where the MRI generates a mix of fluctuations propagating towards and away from the disk midplane. 


\subsection{The mean-square $A W$ amplitude at the coronal base $M_{+\mathrm{b}}$}

In the $\alpha$-disk model (Novikov \& Thorne 1973; Shakura \& Sunyaev 1973), angular momentum transport can be viewed as arising from a turbulent viscosity

$$
v_{\mathrm{t}} \sim v_{\mathrm{T}} l \sim \alpha c_{\mathrm{s}, \mathrm{d}} H
$$

where $v_{\mathrm{T}}$ is the r.m.s. amplitude of the turbulent velocity fluctuations in the disk, $l$ is the correlation length of these velocity fluctuations, $\alpha$ is a dimensionless constant,

$$
H \sim \frac{c_{\mathrm{s}, \mathrm{d}}}{\Omega}
$$

is the disk thickness, $\Omega$ is the angular velocity of the disk,

$$
c_{\mathrm{s}}=\sqrt{\frac{p+p_{\text {rad }}}{\rho}}
$$

is the sound speed and the $d$ subscript in (4.2) and (4.3) indicates that $c_{\mathrm{s}}$ is evaluated at the disk midplane. Nauman \& Blackman (2015) carried out local shearing-box simulations and found that, for Keplerian shear, the correlation time of MRI-generated disk turbulence is $\simeq 0.1(2 \pi / \Omega) \sim \Omega^{-1}$. This correlation time is also comparable to the eddy turnover time in the disk, $l / v_{\mathrm{T}}$, and thus we set

$$
\frac{l}{v_{\mathrm{T}}} \sim \Omega^{-1} .
$$

Dividing the second relation in (4.2) by (4.5) and using (4.3) to eliminate $H$, one obtains (Blackman \& Tan 2004)

$$
v_{\mathrm{T}}^{2} \sim \alpha c_{\mathrm{s}, \mathrm{d}}^{2}
$$

Since the mean-square velocity fluctuation is continuous across the disk/corona boundary, equations (2.28) and (4.6) imply that

$$
M_{+b} \sim \alpha c_{\mathrm{s}, \mathrm{d}}^{2} .
$$

Equation (4.7), in conjunction with (2.33), (2.34), (3.8) and (3.9), can be used to determine the approximate mean-square AW amplitude and heating rate at all positions in the corona and outflow, provided the dependence of $v_{\mathrm{A}}^{\nu}, \overline{u^{\nu}}, \bar{\rho}$ and $\overline{\mathcal{E}}$ on position is known.

\subsection{The AW luminosity of a thin accretion disk}

To estimate the AW energy flux from the disk, we consider the disk's low atmosphere, in which the thermal, Alfvén, and poloidal outflow velocities are non-relativistic, $\overline{\mathcal{E}} \simeq \bar{\rho}, u_{\mathrm{p}} \ll v_{\mathrm{Ap}}$, and the rotational velocity is at most trans-relativistic. For simplicity, we neglect corrections from space-time curvature. The AW contribution to the poloidal energy flux averaged over an annulus of radius $r$ and width $\Delta r \ll r$ centred on the disk's spin axis at height $h$ above the coronal base is

$$
F_{\mathrm{AW}} \simeq \bar{\rho}\left\langle\delta u^{2}\right\rangle v_{\mathrm{Ap}} f \simeq \bar{\rho}^{1 / 2}\left\langle\delta u^{2}\right\rangle B_{\mathrm{p}, \mathrm{net}},
$$


where $f$ is the fraction of the annulus that is threaded by open magnetic-field lines (that connect directly to the distant outflow/jet), and

$$
B_{\mathrm{p}, \text { net }}=f B_{\mathrm{p}}
$$

is the azimuthally averaged poloidal magnetic flux per unit area (i.e. the averaged vertical magnetic field) at radius $r$ and height $h .^{1}$ The factor of $f$ in (4.8) arises because we ignore the AW energy flux on magnetic arches or 'closed loops', which are rooted at both ends in the disk. Because each magnetic loop extends only a finite distance into the corona, $f$ is an increasing function of $h$. Since open magnetic-field lines fan out to fill the volume above closed loops, $B_{\mathrm{p}}$ is a decreasing function of $h$. The product $f B_{\mathrm{p}}=B_{\mathrm{p} \text {, net }}$, however, is approximately independent of $h$, because the same amount of magnetic flux passes through each plane parallel to the disk.

In the low atmosphere, the value of $\eta$ in (3.5) is $\sim 1$, and (3.3), (3.4), (3.5) and (3.7) imply that, along a magnetic field line, $v_{\mathrm{Ap}} / u_{\mathrm{p}} \propto \bar{\rho}^{1 / 2}$, and

$$
\chi \propto \bar{\rho}^{1 / 2} .
$$

Although AW energy dissipates in the low corona, we count such dissipated energy as part of the AW energy flux from the disk. To estimate $F_{\mathrm{AW}}$, we thus neglect dissipative losses when determining $\left\langle\delta u^{2}\right\rangle$ in (4.8). Equation (3.6), with $\gamma_{+} \rightarrow 0$, implies that $\left\langle\delta u^{2}\right\rangle \propto 1 / \chi$ along a field line. Combining this scaling with (4.10), we find that $\bar{\rho}^{1 / 2}\left\langle\delta u^{2}\right\rangle$ is constant along magnetic-field lines, and hence approximately independent of $h$ in the low corona. Since $\bar{\rho}^{1 / 2}\left\langle\delta u^{2}\right\rangle$ and $B_{\mathrm{p}, \text { net }}$ are both independent of $h$, our estimate of $F_{\mathrm{AW}}$ is insensitive to the exact height at which we evaluate $(4.8) .^{2}$ At the coronal base, equation (4.8) can be written, with the aid of (2.28) and (4.7), as

$$
F_{\mathrm{AW}} \sim \rho_{\mathrm{b}}^{1 / 2} \alpha c_{\mathrm{s}, \mathrm{d}}^{2} B_{\mathrm{p}, \text { net }},
$$

where $\rho_{\mathrm{b}}$ is the density at the coronal base. In the $\alpha$-disk model, the radiative flux from the disk is

$$
q \sim \alpha \rho_{\mathrm{d}} c_{\mathrm{s}, \mathrm{d}}^{3},
$$

where $\rho_{\mathrm{d}}$ is the midplane density. Upon dividing (4.11) by (4.12), we obtain

$$
\frac{F_{\mathrm{AW}}}{q} \sim\left(\frac{\rho_{\mathrm{b}}}{\rho_{\mathrm{d}}}\right)^{1 / 2} \beta_{\mathrm{net}, \mathrm{d}}^{-1 / 2}
$$

where

$$
\beta_{\text {net }}=\frac{2\left(p+p_{\text {rad }}\right)}{B_{\mathrm{p}, \text { net }}^{2}},
$$

and $\beta_{\text {net,d }}$ is the value of $\beta_{\text {net }}$ at the disk midplane. All quantities in (4.13) are functions of distance $r$ from the central compact object. Since $q$ peaks at small $r$, the ratio of the disk's AW luminosity to its radiative luminosity is approximately equal to the right-hand side of (4.13) evaluated near the disk's inner edge. ${ }^{3}$

\footnotetext{
${ }^{1}$ The magnetic flux through an individual annulus that arises from closed magnetic loops need not vanish, because loops can connect one annulus to another. Nevertheless, we ignore the magnetic flux associated with closed loops because they contribute zero net flux through the disk as a whole.

${ }^{2}$ AWs do lose energy as the wave-pressure force does work on the outflowing plasma, but the foregoing arguments show that this energy-loss mechanism is negligible in the low atmosphere.

${ }^{3}$ Here, we have assumed that the right-hand side of (4.13) increases as $r$ decreases or depends more weakly on $r$ than does $q$.
} 
Although (4.13) in principle determines $F_{\mathrm{AW}}$, the factors on the right-hand side of (4.13) have large uncertainties. In numerical simulations of disks, $\rho_{\mathrm{b}} / \rho_{\mathrm{d}}$ ranges from $\simeq 10^{-2}$ to $\simeq 0.5$, depending on a number of factors, including whether radiation pressure dominates over plasma pressure and whether the simulation is local or global (e.g. Jiang, Stone \& Davis 2014; Jiang, Davis \& Stone 2016; Zhu \& Stone 2017). The value of $\beta_{\text {net,d }}$ depends on the efficiency with which a disk accretes poloidal magnetic flux. A number of studies have argued that if the magnetic field is passively transported by turbulence in the disk and the turbulent diffusion coefficient is uniform, then the time scale for poloidal flux to diffuse outward is shorter than the accretion time scale of the matter (e.g. van Ballegooijen 1989; Livio, Ogilvie \& Pringle 1999; Guan \& Gammie 2009; Guilet \& Ogilvie 2013). In this case, little poloidal flux accumulates near the central object, and $\beta_{\text {net,d }}$ is extremely large. On the other hand, poloidal flux may be dragged inwards much more efficiently if the turbulent diffusion coefficient becomes small near the boundary between the disk and its atmosphere, if the vertical magnetic field in the disk is concentrated into bundles with a small volume filling factor, or if the vertical field exerts significant torque on the disk material (Livio et al. 1999; Spruit \& Uzdensky 2005; Guilet \& Ogilvie 2013). In numerical simulations, if the initial magnetic field is very weak or has toroidally shaped flux surfaces with comparatively small major radii $r_{i}$, then little poloidal magnetic flux builds up near the central object (see, e.g. De Villiers, Hawley \& Krolik 2003b; Beckwith, Hawley \& Krolik 2008; McKinney, Tchekhovskoy \& Blandford 2012). In contrast, if the initial field is sufficiently strong and $r_{\mathrm{i}}$ sufficiently large, or if substantial poloidal magnetic flux is continuously injected into the simulation domain, then poloidal magnetic flux is dragged inwards so efficiently that the outward magnetic pressure force on disk material becomes comparable to the gravitational force, leading to a magnetically arrested disk (Igumenshchev, Narayan \& Abramowicz 2003; Tchekhovskoy et al. 2011).

We note that the ability of turbulent heating to produce a hot corona starting at the $\beta_{\text {total }}=1$ surface depends not only on $F_{\mathrm{AW}}$, but also on the optical depth at this surface, $\tau_{\mathrm{b}}$. If $\tau_{\mathrm{b}} \gg 1$, and if radiative transfer dominates the vertical energy flux above the $\beta_{\text {total }}=1$ surface, then the temperature must be a decreasing function of distance from the disk midplane in order to drive an outward radiative energy flux (see, e.g. simulation B of Jiang et al. 2014).

\subsection{Self-consistency of the RMHD and $\alpha$-disk approximations}

One of the assumptions underlying the reduced MHD analysis of $\S \S 2.3-2.4$ is the inequality

$$
\frac{\left\langle\delta u^{2}\right\rangle}{v_{\mathrm{A}}^{2}} \ll 1 .
$$

Close to the coronal base, $v_{\mathrm{A}}$ increases with distance from the disk, and we can use (2.28) and (3.8) to rewrite (4.15) as

$$
\left(\frac{M_{+\mathrm{b}}}{v_{\mathrm{Ab}}^{2}}\right)\left(\frac{\chi_{\mathrm{b}}}{\chi}\right)\left(\frac{v_{\mathrm{Ab}}}{v_{\mathrm{A}}}\right)^{3} \ll 1 .
$$

Given (2.28) and (4.7),

$$
\frac{M_{+\mathrm{b}}}{v_{\mathrm{Ab}}^{2}} \sim \frac{\alpha c_{\mathrm{s}, \mathrm{d}}^{2}}{B_{\mathrm{b}}^{2} / \rho_{\mathrm{b}}} \sim \alpha \beta_{\text {total }, \mathrm{d}}\left(\frac{\rho_{\mathrm{b}}}{\rho_{\mathrm{d}}}\right)
$$


where $B_{\mathrm{b}}$ is the magnetic-field strength at the coronal base, and $\beta_{\text {total,d }}$ is the value of $\beta_{\text {total }}$ at the disk midplane. In writing the second order-of-magnitude relationship in (4.17), we have taken the magnetic-field strength to be fairly uniform across the vertical profile of a disk (see, e.g. figure 6 of Beckwith et al. (2008) or figure 2 of Jiang et al. (2014)). The factor of $\alpha \beta_{\text {total,d }}$ in (4.17) is typically one over a few (Blackman, Penna \& Varnière 2008; Sorathia et al. 2012; Hawley et al. 2013). Since $\rho_{b} / \rho_{\mathrm{d}}$ is also less than 1 (or $\left.\ll 1\right), M_{+\mathrm{b}} / v_{\mathrm{Ab}}^{2}$ is smaller than 1 , and (4.16) is at least marginally satisfied at the coronal base. In the low corona, where the flows are at most trans-relativistic and $\overline{\mathcal{E}} \sim \bar{\rho}$, equation (4.10) implies that

$$
\left(\frac{\chi_{\mathrm{b}}}{\chi}\right)\left(\frac{v_{\mathrm{Ab}}}{v_{\mathrm{A}}}\right)^{3} \sim \frac{\bar{\rho}}{\rho_{\mathrm{b}}}\left(\frac{B_{\mathrm{b}}}{\bar{B}}\right)^{3} .
$$

Because the density decreases rapidly with increasing distance $h$ from the coronal base in the low disk atmosphere, we expect the right-hand side of (4.18) to decrease with increasing $h$. Thus, not only is (4.16) at least marginally satisfied at the coronal base, but it becomes a better approximation in the low corona. It is possible that the RMHD approximation breaks down at sufficiently large distances from the disk, but whether this happens depends upon the spatial profiles of the background flow and magnetic field. These profiles, in turn, depend upon how much poloidal magnetic flux is accreted towards the central compact object, which, as discussed above, is uncertain.

The results of this section are also based upon the $\alpha$-disk model, in which disk turbulence is the only mechanism for transporting angular momentum away from the central object, and radiation is the only means by which energy escapes from the disk surface. If poloidal magnetic flux is accreted efficiently towards the central compact object and the disk drives a powerful outflow, then the angular momentum flux and energy flux associated with this outflow could modify the disk structure. Our use of the $\alpha$-disk model thus becomes problematic in the most interesting parameter regime for AW heating, in which $\beta_{\text {net, }}$ is not much greater than 1. If astrophysical disks reach this regime, further work will be needed to model the disks, winds and AW turbulence self-consistently.

\section{A sub-grid model for incorporating reflection-driven Alfvén-wave turbulence into numerical simulations of the averaged GRMHD equations}

The correlation length of the turbulent fluctuations in a thin accretion disk is smaller than the disk's thickness. As a consequence, the AWs launched into the corona of a thin disk have a correlation length perpendicular to the magnetic field, as measured in the AFRF in the low atmosphere of the disk, that is smaller than the disk thickness. These AWs cascade to much smaller scales before dissipating and heating the plasma. In order to carry out a direct numerical simulation of the AW turbulence launched from a thin disk into its corona and outflow, it would be necessary to resolve, within the disk's corona and outflow, length scales much smaller than the disk's thickness, which is unfeasible even on today's fastest supercomputers. An alternative approach is to average the GRMHD equations in the manner described in $\$ 2.2$, solve these averaged GRMHD equations numerically, and incorporate the mean-square AW amplitude as an additional variable that evolves according to (2.30) and (2.33). In this section, we derive a set of averaged GRMHD equations that can be used in this way. ${ }^{4}$ Throughout this section, we make use of the RMHD orderings of $\S 2.3$ and neglect fluctuations in $\rho, u$ (the internal energy) and $p$.

\footnotetext{
${ }^{4} \mathrm{An}$ analogous approach has been used in non-relativistic simulations of the solar wind (Chandran et al. 2011; van der Holst et al. 2014; Usmanov et al. 2014).
} 
To begin, we average (2.7) to obtain $\overline{u^{\mu}} \overline{u_{\mu}}=-\left(1+\left\langle\delta u^{2}\right\rangle\right)$. Since $\overline{u^{\mu}} \overline{u_{\mu}} \neq-1, \overline{u^{\mu}}$ is not a 4-velocity. On the other hand,

$$
u_{0}^{\mu}=\frac{\overline{u^{\mu}}}{\sqrt{1+\left\langle\delta u^{2}\right\rangle}}
$$

is the 4-velocity of the AFRF, since its spatial components (like those of $\overline{u^{\mu}}$ ) vanish in the AFRF, and since

$$
u_{0}^{\mu} u_{0 \mu}=-1 \text {. }
$$

The mass density of the fluid, as measured by an observer with 4-velocity $s^{\mu}$, is $\Gamma^{\mu} s_{\mu}$, where $\Gamma^{\mu}=\rho u^{\mu}$ is the mass-flux 4-vector. The averaged mass density measured by an observer at rest in the AFRF is thus $\rho_{0}=\left\langle\Gamma^{\mu} u_{0 \mu}\right\rangle$, or, equivalently,

$$
\rho_{0}=\rho \sqrt{1+\left\langle\delta u^{2}\right\rangle}
$$

This mass density is larger than the rest-frame density $\rho$ because the fluid moves with respect to the AFRF, and Lorentz contraction causes the apparent density of a moving fluid to be larger than the rest-frame density.

The magnetic field in the frame of an observer $O$ with 4-velocity $s^{\mu}$ is $B_{(s)}^{\mu}=$ $-\left(F^{* \mu \nu}\right) s_{v}$, where $\left(F^{* \mu \nu}\right)=(1 / 2) \epsilon^{\mu \nu \kappa \tau} F_{\kappa \tau}=b^{\mu} u^{v}-b^{v} u^{\mu}$ is the dual of $F^{\mu \nu}$. More precisely, $B_{(s)}^{\mu}$ is that 4-vector which, in the frame of observer $\mathrm{O}$, has a vanishing time component and spatial components equal to the magnetic field that would be measured by $\mathrm{O}$. For example, the magnetic field in the fluid frame is $-\left(F^{* \mu v}\right) u_{v}=b^{\mu}$. In the case that $g^{t t}=-1$, the 4 -velocity $s^{\mu}$ of an observer moving normal to a $t=$ constant 'slice' satisfies $s_{t}=-1$ and $s_{i}=0$, and the magnetic field in the frame of this 'normal observer' is $-\left(F^{* \mu \nu}\right) s_{v}=\left(F^{* \mu t}\right)=b^{\mu} u^{t}-u^{\mu} b^{t} \equiv B^{\mu}$ (Duez et al. 2005). ${ }^{5}$ The spatial components of $B^{\mu}$ were given in (2.9), and $B^{t}$ vanishes since $F^{* \mu \nu}$ is antisymmetric. The averaged magnetic field in the AFRF is $b_{0}^{\mu}=\left\langle-\left(F^{* \mu \nu}\right) u_{0 v}\right\rangle$, or, equivalently,

$$
b_{0}^{\mu}=\overline{b^{\mu}} \sqrt{1+\left\langle\delta u^{2}\right\rangle}-u_{0}^{\mu}\left\langle\delta u^{\alpha} \delta b_{\alpha}\right\rangle .
$$

Averaging (2.8) yields

$$
u_{0}^{\mu} b_{0 \mu}=0 .
$$

The continuity and induction equations for the averaged fluid can be obtained by averaging (2.1) and (2.3) and making use of (5.1), (5.3) and (5.4). This yields

$$
\left(\rho_{0} u_{0}^{v}\right)_{; \nu}=0
$$

and

$$
\left(u_{0}^{\mu} b_{0}^{\nu}-u_{0}^{\nu} b_{0}^{\mu}\right)_{; \nu}=0 .
$$

As in $\$ 2.4$, we restrict our analysis to the case in which AWs travelling away from the disk have much larger amplitudes than AWs traveling toward the disk in the AFRF. However, in contrast to $\S 2.4$, we here allow either $M_{+}$or $M_{-}$to correspond to

\footnotetext{
${ }^{5}$ If $g^{t t} \neq-1$, then the 4-velocity $s^{\mu}$ of an observer moving normal to a $t=$ constant hypersurface satisfies $s_{t}=$ $-1 / \sqrt{-g^{t t}}$ and $s_{i}=0$. The magnetic field in the frame of this normal observer is then $\left(b^{\mu} u^{t}-u^{\mu} b^{t}\right) / \sqrt{-g^{t t}}=$ $B^{\mu} / \sqrt{-g^{t t}}$.
} 
outward-propagating AWs, since in general $\overline{B^{i}}$ points toward the disk in some regions and away from the disk in others. We then set

$$
\delta u^{\mu}=\mp \frac{\delta b^{\mu}}{\mathcal{\mathcal { E }}^{1 / 2}},
$$

where, here and throughout the remainder of this section, the upper sign corresponds to $M_{+} \gg M_{-}$and the lower sign corresponds to $M_{-} \gg M_{+}$. Equation (2.28) then becomes

$$
\left\langle\delta u^{2}\right\rangle=\frac{M_{ \pm}}{4} .
$$

Upon averaging $T^{\mu \nu}$, making use of the RMHD orderings described in $\S 2.3$ and dropping terms $\ll \epsilon^{2} v_{\mathrm{A}}^{2} \overline{\mathcal{E}}$, we obtain

$$
\left\langle T^{\mu \nu}\right\rangle=T_{0}^{\mu \nu}+T_{2}^{\mu \nu}
$$

where

$$
\begin{gathered}
T_{0}^{\mu \nu}=\mathcal{E}_{0} u_{0}^{\mu} u_{0}^{\nu}+\left(p+\frac{b_{0}^{2}}{2}\right) g^{\mu \nu}-b_{0}^{\mu} b_{0}^{\nu}, \\
T_{2}^{\mu \nu}=\mathcal{E}_{0}\left\langle\delta u^{2}\right\rangle\left[\left(2-v_{\mathrm{A} 0}^{2}-\frac{\rho_{0}}{2 \mathcal{E}_{0}}\right) u_{0}^{\mu} u_{0}^{\nu}+\left(\frac{1-v_{\mathrm{A} 0}^{2}}{2}\right) g^{\mu \nu}+v_{\mathrm{A} 0}^{\mu} v_{\mathrm{A} 0}^{\nu} \pm u_{0}^{\mu} v_{\mathrm{A} 0}^{\nu} \pm u_{0}^{\nu} v_{\mathrm{A} 0}^{\mu}\right]
\end{gathered}
$$

and

$$
\mathcal{E}_{0} \equiv \rho_{0}+u+p+b_{0}^{2} \quad v_{\mathrm{A} 0}^{\mu}=b_{0}^{\mu} / \mathcal{E}_{0}^{1 / 2}
$$

The average of (2.2), combined with (5.10), yields

$$
T_{0 ; \nu}^{\mu \nu}=-T_{2 ; \nu}^{\mu \nu} \text {. }
$$

Equations (2.30), (2.33), (5.5), (5.6), (5.7) and (5.14), along with an equation of state $p=p(u, \rho)$, can be solved for $\rho_{0}, u_{0}^{\mu}, b_{0}^{\mu}, u, p$ and $M_{ \pm}$(with the proviso that if $M_{-} \gg M_{+}$, then the plus and minus subscripts need to be interchanged in (2.30) and (2.33)). In this closed system of equations, the AW fluctuations are treated like a fluid that co-evolves with the plasma, and all quantities, including $M_{ \pm}$, vary on the length scale $L$ of the background flow, which greatly exceeds the perpendicular correlation length $\lambda$ of the fluctuations. The effects of turbulent heating and momentum deposition are captured by the source term $-T_{2}^{\mu \nu} ; v$ on the right-hand side of (5.14). Since this source term contains time derivatives, some care is needed when adding it to a GRMHD code. The development of an appropriate numerical algorithm, however, lies beyond the scope of this paper.

If we ignore, for the moment, dissipation of AW turbulence and consider the ideal GRMHD equations, contracting (2.2) with $u_{\mu}$ yields $\left(u^{v} u\right)_{; v}+p u_{; v}^{v}=0$, which implies that the specific entropy of each fluid element is an invariant (Anile 1989). For example, if $p$ were simply $(\gamma-1) u$ for some constant $\gamma$, then $u^{v} \partial_{\nu} \ln \left(p / \rho^{\gamma}\right)=0$ (Del Zanna et al. 2007). However, the dissipation of AW turbulence, which is modelled by the $-2 \gamma_{+} M_{+}$term on the right-hand side of (2.30), should lead to entropy production, i.e. heating. To see how such heating results from (5.14), we contract (5.14) with $\overline{u_{\mu}}$, add (2.30) times $\mathcal{E}_{0} / 4$, and drop terms that are $\ll \epsilon^{2} \mathcal{E} v_{A}^{3} / L$, obtaining

$$
\left(\overline{u^{v}} u\right)_{; \nu}+p \overline{u^{v}} ;=\frac{\gamma_{ \pm} \overline{\mathcal{E}} M_{ \pm}}{2},
$$


where it is $\overline{u^{v}}$ rather than $u_{0}^{v}$ that appears in (5.15). (The $\overline{\mathcal{E}}$ on the right-hand side is interchangeable with $\mathcal{E}_{0}$ to the order of accuracy of the equation.) We identify the right-hand side of (5.15) as the turbulent heating rate, which was previously stated in (2.34) for the case $M_{+} \gg M_{-}$.

\section{Summary and conclusion}

We investigate the propagation, reflection and nonlinear evolution of AWs launched from a turbulent accretion disk. We focus on open-field regions, in which the magnetic-field lines extend from the disk's surface, through the corona and into an overlying outflow. Working within the framework of GRMHD, we derive a set of equations that can be solved for the mean-square AW amplitude and turbulent heating rate as functions of position, and we solve these equations analytically for the case of a time-independent and axisymmetric background flow. Applying these results to the corona and outflow above a thin $\alpha$-disk, we show that the AW energy flux from the disk is approximately $\left(\rho_{\mathrm{b}} / \rho_{\mathrm{d}}\right)^{1 / 2} \beta_{\text {net,d }}^{-1 / 2}$ times the disk's radiative flux, where $\rho_{\mathrm{b}}$ and $\rho_{\mathrm{d}}$ are the densities at the coronal base and disk midplane, respectively, and $\beta_{\text {net,d }}$ is the ratio (evaluated at the disk midplane) of plasma-plus-radiation pressure to the pressure of the average vertical magnetic field. We also derive a set of averaged GRMHD equations that describe the evolution of the background flow in the presence of reflection-driven AW turbulence.

A general feature of reflection-driven AW turbulence is that, as AWs propagate away from the disk into regions with varying values of $v_{\mathrm{A}}$, a significant fraction of the AW energy cascades and dissipates each time $v_{\mathrm{A}}$ changes by a factor of $\sim 2$. As a consequence, much of the AW energy launched by an accretion disk cascades and dissipates within a few Alfvén-speed scale heights of the disk. This makes AW turbulence a promising mechanism for explaining the compact X-ray emitting coronae that are observed around a number of luminous AGN.

In addition to generating compact AGN coronae, AW heating could have a number of consequences for astrophysical disks, coronae and jets. AW heating increases the density scale height in a disk's atmosphere, which enhances the mass outflow rate from the disk and reduces the near-BH mass supply. By loading more mass onto the magnetic-field lines above an accretion disk, AW heating could increase the mechanical luminosity of outflows driven by large-scale magnetic forces. If AW heating deposits substantial energy into the outflowing material far from the disk, this heating could lead to faster outflows (cf. Leer \& Holzer 1980). If AWs in the corona (where $\beta_{\text {total }} \ll 1$ ) mostly heat electrons (Quataert 1998; Quataert \& Gruzinov 1999; Howes 2010; Ressler et al. 2015, 2017), then AW dissipation could lead to substantial coronal emission. Particle-in-cell simulations show that turbulence in relativistic pair plasmas can lead to a power-law tail in the particle energy distribution (Zhdankin et al. 2017), which raises the possibility that AW turbulence in disk coronae and outflows could be an important source of energetic particles. Thermal conduction from the corona into a thin disk may in some cases lead to the evaporation of the disk (Meyer \& Meyer-Hofmeister 1994) and the long-sought 'soft-to-hard' state transition, in which a thin disk inflates, becoming a thick disk. Conversely, if the density of a thick disk of thickness $H \sim c_{\mathrm{s}, \mathrm{d}} / \Omega$ increases sufficiently that radiative cooling causes the disk to collapse parallel to the spin axis to form a thin disk, then $\beta_{\text {net, } \mathrm{d}} \propto \rho_{\mathrm{d}} c_{\mathrm{s}, \mathrm{d}}^{2} / B_{\mathrm{p}, \text { net }}^{2} \propto H / B_{\mathrm{p}, \text { net }}^{2}$ decreases during the collapse (since neither $B_{\mathrm{p}, \text { net }}$ nor $\rho_{\mathrm{d}} H$ changes during the collapse), and there may be a transient period (terminated, e.g. by outward diffusion of poloidal magnetic flux) in which the AW luminosity is strongly enhanced. 
Finally, we note that in current numerical simulations of thin disks, AW turbulence on length scales $<H$ is under-resolved and significantly modified by numerical dissipation. Moreover, since disk coronae are nearly collisionless, the AW energy that is dissipated in a disk's corona is partitioned between electrons and protons in a way that cannot be determined within the framework of MHD. Further studies aimed at capturing the AW heating process and its differential effects on protons and electrons, either analytically or through the use of sub-grid models in numerical simulations, will be needed in order to determine the impact of AW heating on disk winds and jets.

\section{Acknowledgements}

We thank E. Blackman for helpful discussions about turbulence in $\alpha$ disks and the anonymous referee for comments that led to improvements in the manuscript. This work was supported in part by NASA grants NNX15AI80, NNX16AG81G and NNX17AI18G and NSF grant PHY-1500041. F.F.'s participation in this work was supported by NASA through Einstein Postdoctoral Fellowship grant number PF4-150122 awarded by the Chandra X-ray Center, which is operated by the Smithsonian Astrophysical Observatory for NASA under contract NAS8-03060. A.T.'s participation in this work was supported by a TAC Fellowship at the University of California, Berkeley.

\section{Appendix A. The statistically steady, axisymmetric case}

In this appendix, we derive (3.6) and (3.7) starting from (2.30) under the assumption that the background flow is time independent and axisymmetric. Given this assumption, the average of (2.1) leads to

$$
{\overline{u^{v}}}_{; \nu}=-\overline{u^{v}} \partial_{\nu} \ln \bar{\rho}=-u_{\mathrm{p}}^{i} \partial_{i} \ln \bar{\rho},
$$

where we have neglected density fluctuations because of the reduced-MHD orderings in (2.18). With the use of (2.11), (3.2), (3.3) and (3.4), we obtain

$$
\overline{v_{\mathrm{A} ; \nu}^{v}}=\frac{1}{\sqrt{-g}} \partial_{i}\left(\frac{\sqrt{-g} \eta B_{\mathrm{p}}^{i}}{\overline{\mathcal{E}}^{1 / 2}}\right)=B_{\mathrm{p}}^{i} \partial_{i}\left(\frac{\eta}{\overline{\mathcal{E}}^{1 / 2}}\right)=y u_{\mathrm{p}}^{i}\left(\partial_{i} \ln \eta-\frac{1}{2} \partial_{i} \ln \overline{\mathcal{E}}\right) .
$$

Equations (3.4), (A 1) and (A 2) allow us to rewrite (2.30) in the form

$$
(1+y) u_{\mathrm{p}}^{i} \partial_{i} \ln M_{+}+u_{\mathrm{p}}^{i}\left[-2 \partial_{i} \ln \bar{\rho}+\left(\frac{3}{2}+\frac{y}{2}\right) \partial_{i} \ln \overline{\mathcal{E}}+2 y \partial_{i} \ln \eta\right]=-2 \gamma_{+} .
$$

To solve (A 3), we search for an integrating factor $\chi$ that satisfies the equation

$$
(1+y) u_{\mathrm{p}}^{i} \partial_{i} \ln \chi=u_{\mathrm{p}}^{i}\left[-2 \partial_{i} \ln \bar{\rho}+\left(\frac{3}{2}+\frac{y}{2}\right) \partial_{i} \ln \overline{\mathcal{E}}+2 y \partial_{i} \ln \eta\right] .
$$

If we can find such an integrating factor, then we can combine (A 3) and (A 4) and, making use of (3.4), obtain

$$
\left(u_{\mathrm{p}}^{i}+v_{\mathrm{Ap}}^{i}\right) \partial_{i} \ln \left(\chi M_{+}\right)=-2 \gamma_{+},
$$

which is equivalent to (3.6). 
To solve (A 4), we first simplify notation, defining

$$
\begin{aligned}
& A=u_{\mathrm{p}}^{i} \partial_{i} \ln \bar{\rho}, \\
& C=u_{\mathrm{p}}^{i} \partial_{i} \ln \overline{\mathcal{E}},
\end{aligned}
$$

and

$$
D=u_{\mathrm{p}}^{i} \partial_{i} \ln \eta
$$

so that (A 4) becomes

$$
(1+y) u_{\mathrm{p}}^{i} \partial_{i} \ln \chi=-2 A+\left(\frac{3}{2}+\frac{y}{2}\right) C+2 y D .
$$

We note that

$$
u_{\mathrm{p}}^{i} \partial_{i} \ln y=A-\frac{C}{2}+D
$$

and

$$
-2 A+\left(\frac{3}{2}+\frac{y}{2}\right) C+2 y D=\left(A-\frac{C}{2}+D\right)(y-1)+(-A+C+D)(y+1) .
$$

Substituting (A 10) and (A 11) into (A 9) and dividing by $y+1$, we obtain

$$
u_{\mathrm{p}}^{i} \partial_{i} \ln \chi=\left(\frac{y-1}{y+1}\right) u_{\mathrm{p}}^{i} \partial_{i} \ln y-A+C+D,
$$

or, equivalently,

$$
u_{\mathrm{p}}^{i} \partial_{i} \ln \chi=u_{\mathrm{p}}^{i} \partial_{i} \ln \left[\frac{(y+1)^{2}}{y}\right]-u_{\mathrm{p}}^{i} \partial_{i} \ln \bar{\rho}+u_{\mathrm{p}}^{i} \partial_{i} \ln \overline{\mathcal{E}}+u_{\mathrm{p}}^{i} \partial_{i} \ln \eta .
$$

Equation (A 13) can be immediately integrated to yield

$$
\chi=\psi \times \frac{(y+1)^{2} \overline{\mathcal{E}} \eta}{y \bar{\rho}},
$$

where $\psi$ is any quantity that is constant along the lines of flow and force:

$$
u_{\mathrm{p}}^{i} \partial_{i} \psi=\frac{B_{\mathrm{p}}^{i} \partial_{i} \psi}{\kappa}=0 .
$$

We set $\psi=1 / \kappa \bar{\rho}$, which satisfies (A 15) given (3.3). Equations (3.4) and (3.5) then enable us to set $\psi \overline{\mathcal{E}} \eta /(y \bar{\rho})=\overline{\mathcal{E}}^{3 / 2} / \bar{\rho}^{2}$ and $(1+y)^{2}=\left(u_{\mathrm{p}}+v_{\mathrm{Ap}}\right)^{2} / u_{\mathrm{p}}^{2}$ in (A 14), which yields (3.7).

\section{REFERENCES}

Aвbоtт, B. P., Аввотt, R., Аввотt, T. D., Abernathy, M. R., Acernese, F., Ackley, K., Adams, C., Adams, T., Addesso, P., Adhikari, R. X. et al. 2016 Observation of gravitational waves from a binary black hole merger. Phys. Rev. Lett. 116 (6), 061102.

Anile, A. M. 1989 Relativistic Fluids and Magneto-Fluids: With Applications in Astrophysics and Plasma Physics. 
Barnes, A. 1966 Collisionless damping of hydromagnetic waves. Phys. Fluids 9, 1483-1495.

Bavassano, B., Pietropaolo, E. \& Bruno, R. 2000 On the evolution of outward and inward Alfvénic fluctuations in the polar wind. J. Geophys. Res. 105, 15959-15964.

Beckwith, K., Hawley, J. F. \& Krolik, J. H. 2008 The influence of magnetic field geometry on the evolution of black hole accretion flows: similar disks, drastically different jets. Astrophys. J. 678, 1180-1199.

Blackman, E. G., Penna, R. F. \& VArnière, P. 2008 Empirical relation between angular momentum transport and thermal-to-magnetic pressure ratio in shearing box simulations. N. Astron. 13, 244-251.

Blackman, E. G. \& TAN, J. C. 2004 Coronae and outflows from helical dynamos, compatibility with the MRI, and application to protostellar disks. Astrophys. Space. Sci. 292, 395-406.

Blandford, R. D. \& Begelman, M. C. 1999 On the fate of gas accreting at a low rate on to a black hole. Mon. Not. R. Astron. Soc. 303, L1-L5.

Blandford, R. D. \& ZnAJEK, R. L. 1977 Electromagnetic extraction of energy from Kerr black holes. Mon. Not. R. Astron. Soc. 179, 433-456.

Boyer, R. H. \& Lindquist, R. W. 1967 Maximal analytic extension of the Kerr metric. J. Math. Phys. 8, 265-281.

Chandran, B. D. G., Dennis, T. J., Quataert, E. \& Bale, S. D. 2011 Incorporating kinetic physics into a two-fluid solar-wind model with temperature anisotropy and low-frequency Alfvén-wave turbulence. Astrophys. J. 743, 197.

Chandran, B. D. G. \& Hollweg, J. V. 2009 Alfvén wave reflection and turbulent heating in the solar wind from 1 solar radius to 1 AU: an analytical treatment. Astrophys. J. 707, 1659-1667.

Cranmer, S. R. \& VAN BAllegooijen, A. A. 2005 On the generation, propagation, and reflection of Alfvén waves from the solar photosphere to the distant heliosphere. Astrophys. J. Suppl. 156, 265-293.

Cranmer, S. R., van Ballegooijen, A. A. \& Edgar, R. J. 2007 Self-consistent coronal heating and solar wind acceleration from anisotropic magnetohydrodynamic turbulence. Astrophys. $J$. Suppl. 171, 520-551.

De Pontieu, B., Mcintosh, S. W., Carlsson, M., Hansteen, V. H., Tarbell, T. D., Schrijver, C. J., Title, A. M., Shine, R. A., Tsuneta, S., Katsukawa, Y. et al. 2007 Chromospheric Alfvénic waves strong enough to power the solar wind. Science 318, 1574-1577.

De Villiers, J.-P., Hawley, J. F. \& Krolik, J. H. $2003 a$ Magnetically driven accretion flows in the Kerr metric. I. Models and overall structure. Astrophys. J. 599, 1238-1253.

De Villiers, J.-P., Hawley, J. F. \& Krolik, J. H. $2003 b$ Magnetically driven accretion flows in the Kerr metric. I. Models and overall structure. Astrophys. J. 599, 1238-1253.

Del Zanna, L., Zanotti, O., Bucciantini, N. \& Londrillo, P. 2007 ECHO: a Eulerian conservative high-order scheme for general relativistic magnetohydrodynamics and magnetodynamics. Astron. Astrophys. 473, 11-30.

Dmitruk, P., Matthaeus, W. H., Milano, L. J., Oughton, S., Zank, G. P. \& Mullan, D. J. 2002 Coronal heating distribution due to low-frequency, wave-driven turbulence. Astrophys. J. 575, 571-577.

Duez, M. D., LiU, Y. T., Shapiro, S. L. \& Stephens, B. C. 2005 Relativistic magnetohydrodynamics in dynamical spacetimes: numerical methods and tests. Phys. Rev. D. 72 (2), 024028.

Elsasser, W. M. 1950 The hydromagnetic equations. Phys. Rev. 79, 183-183.

FANAROFF, B. L. \& RiLEY, J. M. 1974 The morphology of extragalactic radio sources of high and low luminosity. Mon. Not. R. Astron. Soc. 167, 31P-36P.

Galeev, A. A., Rosner, R. \& Vaiana, G. S. 1979 Structured coronae of accretion disks. Astrophys. J. 229, 318-326.

Gammie, C. F., McKinney, J. C. \& Tóth, G. 2003 HARM: a numerical scheme for general relativistic magnetohydrodynamics. Astrophys. J. 589, 444-457.

Ghez, A. M., Salim, S., Hornstein, S. D., Tanner, A., Lu, J. R., Morris, M., Becklin, E. E. \& Duchêne, G. 2005 Stellar orbits around the galactic center black hole. Astrophys. $J$. 620, 744-757. 
Guan, X. \& Gammie, C. F. 2009 The turbulent magnetic prandtl number of MHD turbulence in disks. Astrophys. J. 697, 1901-1906.

Guilet, J. \& Ogilvie, G. I. 2013 Transport of magnetic flux and the vertical structure of accretion discs - II. Vertical profile of the diffusion coefficients. Mon. Not. R. Astron. Soc. 430, 822-835.

Hawley, J. F., Richers, S. A., Guan, X. \& KroliK, J. H. 2013 Testing convergence for global accretion disks. Astrophys. J. 772, 102.

Heinemann, M. \& OlberT, S. 1980 Non-WKB Alfven waves in the solar wind. J. Geophys. Res. 85, 1311-1327.

Hollweg, J. V. 1978 Fast wave evanescence in the solar corona. Geophys. Res. Lett. 5, 731-734.

Hollweg, J. V., Cranmer, S. R. \& Chandran, B. D. G. 2010 Coronal Faraday rotation fluctuations and a wave/turbulence-driven model of the solar wind. Astrophys. J. 722, $1495-1503$.

Horbury, T. S., Balogh, A., Forsyth, R. J. \& Smith, E. J. 1995 Anisotropy of inertial range turbulence in the polar heliosphere. Geophys. Res. Lett. 22, 3405-3408.

Howes, G. G. 2010 A prescription for the turbulent heating of astrophysical plasmas. Mon. Not. R. Astron. Soc. 409, L104-L108.

Howes, G. G., Bale, S. D., Klein, K. G., Chen, C. H. K., Salem, C. S. \& TenBarge, J. M. 2012 The slow-mode nature of compressible wave power in solar wind turbulence. Astrophys. J. Lett. 753, L19.

Igumenshchev, I. V., Narayan, R. \& Abramowicz, M. A. 2003 Three-dimensional magnetohydrodynamic simulations of radiatively inefficient accretion flows. Astrophys. J. 592, 1042-1059.

Iroshnikov, P. S. 1963 Turbulence of a conducting fluid in a strong magnetic field. Astron. Zh. 40, 742 .

JiAng, Y.-F., Davis, S. W. \& Stone, J. M. 2016 Iron opacity bump changes the stability and structure of accretion disks in active galactic nuclei. Astrophys. J. 827, 10.

JiAng, Y.-F., Stone, J. M. \& DAVIS, S. W. 2014 Radiation magnetohydrodynamic simulations of the formation of hot accretion disk coronae. Astrophys. J. 784, 169.

Klein, K. G., Howes, G. G., Tenbarge, J. M., Bale, S. D., Chen, C. H. K. \& Salem, C. S. 2012 Using synthetic spacecraft data to interpret compressible fluctuations in solar wind turbulence. Astrophys. J. 755, 159.

Klein, L. W., Roberts, D. A. \& Goldstein, M. L. 1991 Anisotropy and minimum variance directions of solar wind fluctuations in the outer heliosphere. J. Geophys. Res. 96, 3779-3788.

Komissarov, S. S. 1999 Numerical simulations of relativistic magnetized jets. Mon. Not. R. Astron. Soc. 308, 1069-1076.

Kraichnan, R. H. 1965 Inertial-range spectrum of hydromagnetic turbulence. Phys. Fluids 8, 1385.

Leer, E. \& Holzer, T. E. 1980 Energy addition in the solar wind. J. Geophys. Res. 85, 4681-4688.

Livio, M., Ogilvie, G. I. \& Pringle, J. E. 1999 Extracting energy from black holes: the relative importance of the Blandford-Znajek mechanism. Astrophys. J. 512, 100-104.

MARON, J. \& GoldREICH, P. 2001 Simulations of incompressible magnetohydrodynamic turbulence. Astrophys. J. 554, 1175-1196.

McKinney, J. C. \& GAmmie, C. F. 2004 A measurement of the electromagnetic luminosity of a Kerr black hole. Astrophys. J. 611, 977-995.

McKinney, J. C., Tchekhovskoy, A. \& Blandford, R. D. 2012 General relativistic magnetohydrodynamic simulations of magnetically choked accretion flows around black holes. Mon. Not. R. Astron. Soc. 423, 3083-3117.

Mestel, L. 1961 A note on equatorial acceleration in a magnetic star. Mon. Not. R. Astron. Soc. 122, 473.

Meyer, F. \& Meyer-Hofmeister, E. 1994 Accretion disk evaporation by a coronal siphon flow. Astron. Astrophys. 288, 175-182.

Miyoshi, M., Moran, J., Herrnstein, J., Greenhill, L., Nakai, N., Diamond, P. \& Inoue, M. 1995 Evidence for a black hole from high rotation velocities in a sub-parsec region of NGC4258. Nature 373, 127-129.

NARAYAn, R. \& YI, I. 1994 Advection-dominated accretion: a self-similar solution. Astrophys. J. Lett. 428, L13-L16. 
Nauman, F. \& Blackman, E. G. 2015 Sensitivity of the magnetorotational instability to the shear parameter in stratified simulations. Mon. Not. R. Astron. Soc. 446, 2102-2109.

Novikov, I. D. \& Thorne, K. S. 1973 Astrophysics of black holes. In Black Holes (Les Astres Occlus) (ed. C. Dewitt \& B. S. Dewitt), pp. 343-450.

Perez, J. C. \& Chandran, B. D. G. 2013 Direct numerical simulations of reflection-driven, reduced MHD turbulence from the sun to the Alfven critical point. Astrophys. J. 776, 124.

QuATAert, E. 1998 Particle heating by Alfvenic turbulence in hot accretion flows. Astrophys. J. 500, 978.

Quataert, E. \& GRUZinov, A. 1999 Turbulence and particle heating in advection-dominated accretion flows. Astrophys. J. 520, 248-255.

Quataert, E. \& Gruzinov, A. 2000 Convection-dominated accretion flows. Astrophys. J. 539, 809-814.

Reis, R. C. \& Miller, J. M. 2013 On the size and location of the x-ray emitting coronae around black holes. Astrophys. J. Lett. 769, L7.

Ressler, S. M., Tchekhovskoy, A., Quataert, E., Chandra, M. \& Gammie, C. F. 2015 Electron thermodynamics in GRMHD simulations of low-luminosity black hole accretion. Mon. Not. R. Astron. Soc. 454, 1848-1870.

Ressler, S. M., Tchekhovskoy, A., Quataert, E. \& Gammie, C. F. 2017 The disc-jet symbiosis emerges: modelling the emission of Sagittarius $\mathrm{A}^{*}$ with electron thermodynamics. Mon. Not. R. Astron. Soc. 467, 3604-3619.

Schekochihin, A. A., Cowley, S. C., Dorland, W., Hammett, G. W., Howes, G. G., QuATAerT, E. \& TATSuno, T. 2009 Astrophysical gyrokinetics: kinetic and fluid turbulent cascades in magnetized weakly collisional plasmas. Astrophys. J. Suppl. 182, 310-377.

Schekochinin, A. A., Parker, J. T., Highcock, E. G., Dellar, P. J., Dorland, W. \& Hammett, G. W. 2016 Phase mixing versus nonlinear advection in drift-kinetic plasma turbulence. J. Plasma Phys. 82 (2), 905820212.

ShakURA, N. I. \& SUnYAEV, R. A. 1973 Black holes in binary systems. Observational appearance. Astron. Astrophys. 24, 337-355.

Sorathia, K. A., Reynolds, C. S., Stone, J. M. \& Beckwith, K. 2012 Global simulations of accretion disks. I. Convergence and comparisons with local models. Astrophys. J. 749, 189.

Spruit, H. C. \& UzDensky, D. A. 2005 Magnetic flux captured by an accretion disk. Astrophys. J. 629, 960-968.

Tchekhovskoy, A., Narayan, R. \& McKinney, J. C. 2011 Efficient generation of jets from magnetically arrested accretion on a rapidly spinning black hole. Mon. Not. R. Astron. Soc. 418, L79-L83.

TU, C. \& MARSCH, E. 1995 MHD structures, waves and turbulence in the solar wind: observations and theories. Space Sci. Rev. 73, 1-210.

Usmanov, A. V., Goldstein, M. L. \& Matthaeus, W. H. 2014 Three-fluid, three-dimensional magnetohydrodynamic solar wind model with Eddy viscosity and turbulent resistivity. Astrophys. J. 788, 43.

UZDensky, D. A. \& Goodman, J. 2008 Statistical description of a magnetized corona above a turbulent accretion disk. Astrophys. J. 682, 608-629.

VAN Ballegooijen, A. A. 1989 Magnetic fields in the accretion disks of cataclysmic variables. In Accretion Disks and Magnetic Fields in Astrophysics (ed. G. Belvedere), Astrophysics and Space Science Library, vol. 156, pp. 99-106.

van Ballegooijen, A. A. \& Asgari-Targhi, M. 2016 Heating and acceleration of the fast solar wind by Alfvén wave turbulence. Astrophys. J. 821, 106.

VAN BAllegooijen, A. A. \& Asgari-Targhi, M. 2017 Direct and inverse cascades in the acceleration region of the fast solar wind. Astrophys. J. 835, 10.

van der Holst, B., Sokolov, I. V., Meng, X., Jin, M., Manchester IV, W. B., Tóth, G. \& Gombosi, T. I. 2014 Alfvén wave solar model (AWSoM): coronal heating. Astrophys. J. 782, 81.

VelLI, M. 1993 On the propagation of ideal, linear Alfven waves in radially stratified stellar atmospheres and winds. Astron. Astrophys. 270, 304-314. 
Verdini, A. \& Velli, M. 2007 Alfvén waves and turbulence in the solar atmosphere and solar wind. Astrophys. J. 662, 669-676.

Verdini, A., Velli, M., Matthaeus, W. H., Oughton, S. \& Dmitruk, P. 2010 A turbulencedriven model for heating and acceleration of the fast wind in coronal holes. Astrophys. J. Lett. 708, L116-L120.

Yao, S., He, J.-S., Marsch, E., Tu, C.-Y., Pedersen, A., Rème, H. \& Trotignon, J. G. 2011 Multi-scale anti-correlation between electron density and magnetic field strength in the solar wind. Astrophys. J. 728, 146.

Zhdankin, V., Werner, G. R., Uzdensky, D. A. \& Begelman, M. C. 2017 Kinetic turbulence in relativistic plasma: from thermal bath to nonthermal continuum. Phys. Rev. Lett. 118 (5), 055103.

Zhu, Z. \& Stone, J. M. 2017 Global evolution of an accretion disk with net vertical field: coronal accretion, flux transport, and disk winds. ArXiv e-prints, arXiv:1701.04627. 